\title{
Spatio-Temporal Analysis of Highly Pathogenic Avian Influenza outbreaks in Ghana.
}

\author{
Esther N. Dsani ${ }^{1,} 2$ \\ ${ }^{1}$ Veterinary services, Ministry of Food and Agriculture, Ghana, Accra, Greater Accra, Ghana, ${ }^{2}$ School of Public Health, University of Ghana, Accra, \\ Ghana
}

\section{Objective}

The purpose of the study was to characterize the spatial distribution and temporal patterns of laboratory confirmed H5N1 outbreaks from January 2007 to December 2017 in Ghana.

\section{Introduction}

Highly pathogenic avian influenza (HPAI) subtype H5N1 virus causes a highly contagious disease in poultry with up to $100 \%$ mortality and occasionally causes sporadic human infection. The first outbreak of HPAI H5N1 in Africa was reported in Nigeria in 2006 and has since been reported in seven other African countries with confirmed human cases and outbreaks in poultry. Since the emergence of Highly Pathogenic Avian Influenza (HPAI), virus subtype H5N1 in Ghana in 2007, outbreaks in poultry have led to dire economic consequences for the poultry sector, resulting from mass destruction of affected flocks. An economy heavily dependent on agriculture, the persistence of outbreaks threaten the livelihood of farmers who depend on poultry production for survival.

Despite significant efforts made in HPAI-H5N1 control and prevention in Ghana, outbreaks persist and continue to spread to new areas. It is uncertain to what extent different pathways contribute to the introduction and the dissemination of the virus in Ghana. There is a need to understand the complex nature of the interactions between local and migratory fowl, the risk of transmission due to human endeavor and trade mechanisms that increase the likelihood of HPAI-H5N1 outbreaks in Ghana.

\section{Methods}

Data for the study was sourced from national outbreak records at the Veterinary Services Directorate.

The study analyzed outbreak data for the years 2007-2017. Data retrieved from outbreak reports included the date of onset of outbreak, location and geographic coordinates, type and number of poultry species affected, natural deaths of birds and type of farming system on outbreak farms. We calculated frequency distributions for the types of poultry species affected, the type of farming system and mortality rates on affected premises.

We described the distribution of HPAI-H5N1 outbreaks using coordinate maps in ArcGIS and displayed relevant sites of waterfowl and wild bird habitation. To describe the temporal pattern of HPAI-H5N1 outbreaks in Ghana for the period, we created an epidemic curve by plotting the monthly number of outbreaks for the period January 2007 to December 2017 in Excel. We used space-time scan statistics to determine significant local clusters.

\section{Results}

A total of sixty-six (66) outbreaks of HPAI-H5N1 occurred in Ghana from January 2007 to December 2017. The outbreak sites were distributed in seven (7) out of ten (10) regions in Ghana. The affected regions are located in the southern and middle belt of Ghana. Most of the outbreaks (74.2\%) occurred in densely populated areas of the Greater Accra region. Overall, layer flocks were mostly affected with $56 \%$ of affected premises constituting layer farms. Commercial farms and backyard farms made up the majority of affected farms (50\% and 42.4\%). Free ranging birds were the least affected farm type (7.6\%). Two epidemic waves were identified for H5N1 in Ghana; the first wave with 6 outbreaks, lasted a period of four (4) months from April to July 2007, and the second with 60 outbreaks, spanned a period of 2 years from April 2015 to November 2016. Temporal distribution of the outbreaks showed that the outbreak peaked in May 2007 for the first wave and in July 2017 for the second wave with minor peaks observed in April and July 2016. The decrease in the number of the outbreaks after July in both waves is attributed to the onset of 
slaughter and trade restrictions for poultry in affected areas. Space-time scan statistics identified significant primary clusters of H5N1 outbreaks in the coastal belt of the Greater Accra region, characterized by major commercial activities and the presence of wetlands of relevance to wild birds and migratory waterfowl.

\section{Conclusions}

Two (2) major waves of H5N1 outbreaks occurred in Ghana between 2007 and 2017. The distribution of outbreaks and poultry species in both waves, show that the epidemiology of H5N1 virus in Ghana is changing. The findings highlight the importance of reviewing existing control and preventive measures as well as strengthening avian influenza surveillance in proposed high- risk areas.

\section{Acknowledgement}

We thank Dr. Helena Acquah and Dr. Fenteng Danso, Veterinary Epidemiologists of the Epidemiology Unit at the Veterinary Services Directorate of Ghana for their support.

\section{References}

Foreign Animal Diseases. Revised 2008 Seventh Edition. Committee on foreign and emerging diseases of the United States Animal Health Association. Avian Influenza, OIE terrestrial manual 2015

To KKW, et al. 2012. Avian influenza A H5N1 virus: a continuous threat to humans. Emerg Microbes Infect. 1(9), e25. PubMed https://doi.org/10.1038/emi.2012.24

Watanabe Y, Ibrahim MS, Suzuki Y, Ikuta K. 2012. The changing nature of avian influenza A virus (H5N1). Trends Microbiol. 20(1), 11-20. PubMed https://doi.org/10.1016/j.tim.2011.10.003 